PTH-117 SEX HORMONE RECEPTOR EXPRESSION IN OESOPHAGEAL ADENOCARCINOMA AND RECURRENCE AND SURVIVAL: A RETROSPECTIVE COHORT STUDY

${ }^{1}$ Una CMc Menamin*, ${ }^{2}$ James Trainor, ${ }^{1}$ Helen G Coleman, ${ }^{2}$ Damian T McManus, ${ }^{3}$ Brian T Johnston, ${ }^{4}$ Richard Turkington. ${ }^{1}$ Centre for Public Health, Queen's University Belfast, Belfast, UK; ${ }^{2}$ Department of Pathology, Belfast Health and Social Care Trust, Belfast, UK; ${ }^{3}$ Department of Gastroenterology, Royal Victoria Hospital, Belfast Health and Social Care Trust, Belfast, UK; ${ }^{4}$ Centre for Cancer Research and Cell Biology, Queen's University Belfast, Belfast, UK

\subsection{6/gutjnl-2018-BSGAbstracts.291}

Introduction The most striking epidemiological feature of Oesophageal adenocarcinoma (OAC) is its strong unexplained male predominance, suggesting a protective effect for oestrogens, but few studies have investigated expression of sex hormone receptors in OAC. In a retrospective cohort of OAC patients, we evaluated Oestrogen Receptor (ER) $\alpha$ and $\beta$ and Androgen Receptor (AR) tumour expression and investigated associations with OAC recurrence and survival.

Methods We identified 148 OAC patients who underwent neo-adjuvant chemotherapy prior to surgical resection between 2004-2012 at the Northern Ireland Cancer Centre. Immunohistochemical expression of $\mathrm{ER} \alpha, \mathrm{ER} \beta$ and AR was scored by two independent observers, blinded to the clinical data. Cox proportional hazards regression was used to calculate hazard ratios (HR) and 95\% confidence intervals (CI) for associations between sex hormone receptor expression and overall survival, cancer-specific survival and recurrence-free survival. All analyses were adjusted for clinic-pathological and lifestyle factors including age at diagnosis, sex, pathological nodal stage, primary site, lymphovascular invasion, circumferential margin involvement, PET response and smoking. Sub-group analysis was conducted by Siewert classification.

Results Weak positive expression was identified for ER $\alpha$ (6/139) and AR (4/138) while moderate positive expression was observed for ER $\beta$ (43/138). After a mean follow-up of 3 years ( $\max 9$ years), no significant associations were observed for $\mathrm{ER} \alpha, \mathrm{ER} \beta$ or AR expression and OAC recurrence or survival. ER $\beta$ expression however was associated with significant improvements in overall survival (HR 0.38, 95\% CI 0.16, 0.88), cancer-specific survival (HR $0.36,95 \%$ CI $0.15,0.84$ ) and recurrence-free survival (HR 0.28, 95\% CI 0.12, 0.69) in patients with adenocarcinoma of the distal oesophagus (Siewert type I).

Conclusion In the largest study to date, we found little evidence of $\mathrm{ER} \alpha$ or AR expression in OAC. We observed moderate expression of ER $\beta$ and suggestive evidence that its expression was associated with reduced recurrence and death in patients with adenocarcinoma of the distal oesophagus. Further studies however are required to replicate our findings to determine if the ER system could be a potential prognostic biomarker in OAC.

\section{PTH-118 HISTOPATHOLOGIST FEATURES PREDICTIVE OF DIAGNOSTIC CONCORDANCE AMONGST AN INTERNATIONAL SAMPLE OF PATHOLOGISTS DIAGNOSING BARRETT'S DYSPLASIA}

\footnotetext{
${ }^{1}$ Helen Coleman*, ${ }^{2,3}$ Myrtle van der Wel, ${ }^{4}$ Marnix Jansen, ${ }^{2}$ Sybren Meijer, BOLERO Study group. 'Centre for Public Health, Queen's University Belfast, Belfast, UK; ${ }^{2}$ Department of Pathology, Academic Medical Center, Amsterdam, The Netherlands; ${ }^{3}$ Department of Gastroenterology, Academic Medical Center, Amsterdam, The Netherlands; ${ }^{4}$ Department of Pathology, University College Hospital, London, UK
}

10.1136/gutjnl-2018-BSGAbstracts.292
Introduction Histopathological diagnosis of dysplasia in Barrett's oesophagus (BO) is the gold standard for patient risk stratification, but is subject to significant interobserver variation. We investigated histopathologist features that predict diagnostic performance amongst a large international cohort of gastrointestinal (GI) pathologists.

Methods An online scoring environment was developed for GI-pathologists $(n=55)$ from over 20 countries to grade a case set of 55 digitalised $\mathrm{BO}$ biopsies encompassing the complete spectrum from non-dysplastic Barrett's oesophagus (NDBO), indefinite, low and high-grade dysplasia (IND/LGD/ HGD). Case interpretations were recorded before and after revealing P53 immunohistochemistry. Detailed histopathologist demographic data (experience, centre volume, fellowship training etc.) was obtained through an online questionnaire. A consensus gold standard diagnosis was obtained for the entire case set through a reference panel of four expert pathologists. Multivariate regression analyses was conducted to identify pathologist predictors of concordance.

Results We recorded over 6000 case diagnoses. Of 2,805 hour and $\mathrm{E}$ diagnoses, we found excellent concordance for NDBO (643 of 816 diagnoses; 79\%) and HGD (544 of 765 diagnoses; $71 \%)$ and intermediate concordance for LGD (382 of $918 ; 42 \%)$ and IND (70 of $306 ; 23 \%)$, replicating known glass slide test characteristics. Major over or under-interpretations (i.e. NDBO overstaged as LGD/HGD, or LGD/HGD understaged as NDBO) were reported in 248 diagnoses $(8.8 \%)$. Addition of p53 staining further improved diagnostic consensus, but had limited impact on major over or underinterpretations. Multivariate regression analyses revealed independent histopathologist predictors of expert level diagnostic performance, including; at least 5 years of experience, working within a teaching hospital, viewing 5-20 Barrett's cases per week, adherence to major guidelines, and an interest in digital pathology.

Conclusions Using this rich dataset representing a heterogeneous group of gastrointestinal pathologists working globally, we have quantified diagnostic performance for BO dysplasia diagnosis using digital case review. Our results reveal predictors of diagnostic performance at expert level, and will aid formulation of quality assurance criteria for guideline development.

\section{PTH-119 INTERNATIONAL MULTICENTRE STUDY OF MALLORY WEISS TEAR RELATED GI BLEEDING: DEMOGRAPHICS, ENDOSCOPIC THERAPY AND OUTCOME}

${ }^{1}$ Lucy Lynch*, ${ }^{2}$ Stig Borbjerg Laursen, ${ }^{3}$ Loren Laine, ${ }^{4}$ Harry Dalton, ${ }^{4}$ Nick Michell, ${ }^{5}$ Jeffery Ngu, ${ }^{6}$ Michael Schultz, ${ }^{1}$ Adrian Stanley. ${ }^{1}$ Glasgow Royal Infirmary; ${ }^{2}$ Odense University Hospital; ${ }^{3}$ Yale-New Haven Hospital; ${ }^{4}$ Royal Cornwall Hospital; ${ }^{5}$ Singapore General Hospital; ${ }^{6}$ Dunedin Hospital

\subsection{6/gutjnl-2018-BSGAbstracts.293}

Introduction Mallory Weiss tears (MWT) are relatively uncommon causes of upper GI bleeding (UGIB) and patients with these lesions are generally considered at low risk of poor outcome. However there are relatively limited data on this condition. In addition, there is uncertainty about which patients with MWT require endoscopic therapy and which modality should be applied. We aimed to describe an international cohort of patients presenting with UGIB secondary to MWT, including the endoscopic therapy undertaken. We also 